\title{
Hybrid Deliberative/Reactive Control of a Scanning System for Landmine Detection
}

\author{
E. Garcia * P. Gonzalez de Santos \\ Automatic Control Department, Industrial Automation Institute (CSIC), 28500 \\ Arganda del Rey, Madrid, Spain
}

\begin{abstract}
Antipersonnel mines infest fields all over the world. According to recent estimates, landmines are killing and maiming more than 2,000 innocent civilians per month. The problem of landmine detection and removal requires the cooperation of a number of engineering fields, which in turn poses a need for new technologies, such as improved sensors, efficient manipulators and mobile robots. This paper describes the configuration and control architecture of a scanning manipulator for detecting antipersonnel landmines. The scanning system is part of a demining system based on a walking robot that acts as the carrier for the scanning manipulator. Broadly speaking, the scanning system consists of a sensor head that can detect certain kinds of landmines and, to move the sensor head over large areas, a manipulator that has been appropriately sensorized to scan irregular terrains in the presence of obstacles. The proposed control architecture is of the hybrid deliberative/reactive type: A deliberative controller defines a sweep trajectory that furnishes complete coverage of the infested area, while two reactive controllers are involved in on-line adaptation to the environment. Experiments show the good performance of the whole system.
\end{abstract}

Key words: Landmine detection, robotics, robot manipulator, deliberative/reactive hybrid control

\section{Introduction}

Detection and removal of antipersonnel landmines is one of the serious problems humankind faces today. According to recent estimates, landmines are killing and maiming more than 2,000 innocent civilians per month. Demining

\footnotetext{
* Corresponding author.

Email address: egarcia@iai.csic.es (E. Garcia).
} 
is currently done by hand, in a procedure that is risky and, above all, slow [1]: It is twenty times faster to infest a field than to clear it. Solutions are being explored in different engineering fields. The best course for releasing human operators from this risky task is to apply fully automatic systems; however, this solution is still far from successful, due to the difficulty of applying fully autonomous systems in such unstructured environments. Nevertheless, there are some aspects of the job that robots can do quite well, like scanning the ground to detect and locate buried mines. For this task, efficient sensors and detectors are required to detect and, if possible, identify different mines [2].

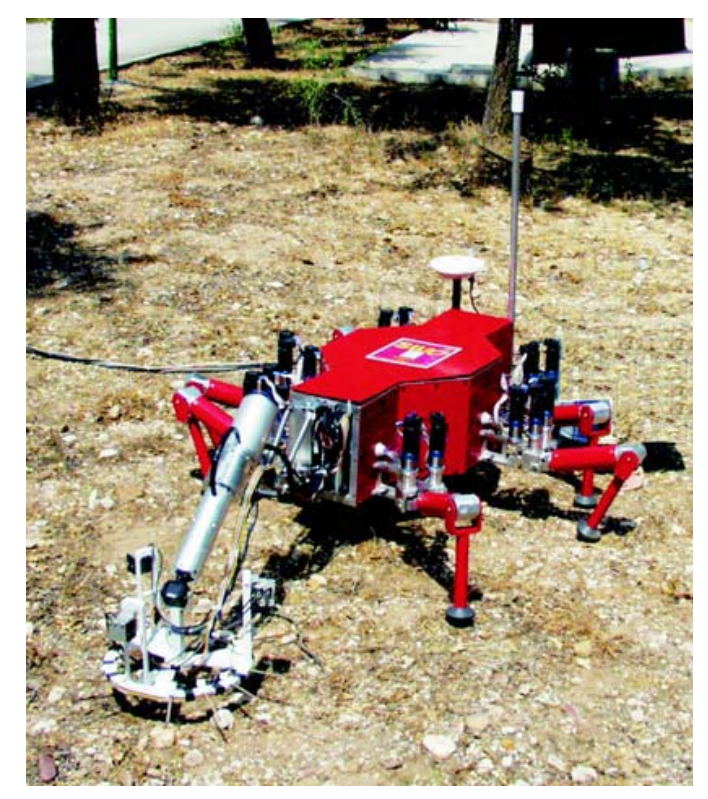

Fig. 1. DYLEMA demining system

Based on previous experience in robotics, the IAI-CSIC has configured the DYLEMA system, based on a legged robot for landmine detection and location (see Fig. 1) [3,4]. DYLEMA is a Spanish acronym that stands for "Efficient Detection and Location of Antipersonnel Landmines." The main aim of the project is to develop a system that integrates relevant technologies in the fields of legged locomotion and sensor systems, in order to identify what is needed in the process.

This paper presents the ongoing results of the scanning manipulator carried by the walking robot. The scanning manipulator is a 5-DOF robot manipulator endowed with a sensor head. The sensor head contains a commercial metal detector to locate buried mines and range sensors used to control the detector's movement as it follows the ground contour. A simple array of bumpers around the sensor head detects obstacles in the scanning trajectory. The on-board controller modifies the search trajectory on-line to avoid obstacles like rocks or trees. Therefore, the scanning system supports the autonomous search for landmines in unstructured, natural terrains. The control architecture herein 
presented is of the deliberative/reactive hybrid type; it thus commands a predefined sweep trajectory that ensures complete coverage of the swept area, yet it modifies the trajectory in a reactive manner to adapt to terrain roughness and avoid obstacles in its path. The complete-coverage sweep motion of the scanning system is original to this project. Use of the proposed reactive controllers enables the system to operate efficiently in natural, unstructured environments without resorting to complex, expensive methods for on-line terrain mapping [5]. In this paper we present the design and control architecture of the scanning system and some experimental results that show the efficient performance of the whole system.

\section{The DYLEMA Project}

The DYLEMA Project, configured around the SILO6 walking robot, has been designed taking into consideration the features required by international organizations such as the UN that demands a clearance efficiency of about $99.6 \%$ for humanitarian purposes. The sensor head of the DYLEMA system is expected to achieve a speed of about $40 \mathrm{~cm} / \mathrm{s}$ in detection mode and $2.5 \mathrm{~cm} / \mathrm{s}$ in identification mode. Mines and potential alarms are located with and accuracy of about $2 \mathrm{~cm}$ by using Global Differential Positioning System (DGPS) and Kalman filter techniques [4], and the terrain inspection speed is about 60 - $400 \mathrm{~m}^{2} /$ day depending on the terrain. Obviously, de-mining rate is low in comparison with that obtained by other kinds of vehicles in simple scenarios. However, our system is specially tailored for difficult areas or scenarios with very difficult access where traditional vehicles cannot work properly or just cannot enter. Similar research projects exist that consider a legged robot as the appropriate mobile platform for demining such difficult areas like irregular grounds or terrains with a large amount of obstacles (trees, big stones, etc), where a wheeled robot cannot operate or may require many different manoeuvres to precede forward.

Most walking robots developed for humanitarian demining activities are based on frames (sliding platforms) [6]. The main disadvantage of the frame-based design is the reduced flexibility of the walking mechanism at selecting footholds, as each frame contains four legs located at fixed positions and only relative motion between frames can be achieved. This is a clear drawback for the envisaged application because the robot is expected to avoid stepping over mines and obstacles. On the contrary, the COMET project by Prof. Nonami is based on a six-legged robot with insect-like legs which allow for foothold selection and navigation in very irregular terrain [7]. The selection of six legs allows for a trade-off between stability and speed. However, the consideration of six legs in the COMET III robot is based only on stability purposes because the robot walks using slow gaits that keep four and five legs in support 
simultaneously. Conversely, the SILO6 robot used in the DYLEMA project exploits the benefits of a six-legged system by using an alternate-tripod gait. Another advantage of our proposed demining system is its small size and weight. The SILO6 robot is $1 \mathrm{~m}$ long and weighs $60 \mathrm{Kg}$, which results in a portable platform really adequate to operate in infested areas of difficult access like narrow bridges and ravines, abrupt valleys or debris areas compared to the COMET III dimensions ( $4 \mathrm{~m}$ long and $1200 \mathrm{Kg}$ weight). Therefore, the DYLEMA project complies with the first requirement for a demining platform to be "small enough to be portable (by manned ground transportation to access the minefield or to be removed from the minefield in case of failure), easy to transport and deploy," as stated by the International Test and Evaluation Program for Humanitarian Demining [8]. Another particularity of the SILO6 robot is the optimization of leg-force distribution intrinsic to its design, which reduces energy consumption, a paramount feature for demining applications. Further details of the SILO6 design and specifications of the DYLEMA project can be found in [4].

To carry out demining tasks, every mine-location project includes a vehiclemounted mine detector. The most proposed sensor technology combines metal detector (MD) and ground penetrating radar (GPR). It has been learned that positioning and orientation control must be improved in scanning the ground with a sensor head in order to make the best use of MDs mounted on vehicles [8]. The most usual way to achieve efficient position and orientation of the sensor head is the use of 5 -DOF robotic systems, three degrees of freedom for position control and two more for ground-profile adaptation. At first it seems that using legged robots could reduce this amount of required degrees of freedom due to the added mobility of the walking vehicle. However, the workspace of a walking robot gets too limited to allow for sensor head orientation. Also, the stability of the vehicle would be a limitation for such purposes. Therefore, the amount of degrees of freedom of the scanning system does not depend on the vehicle's mobility. The COMET III robot uses a 3-DOF scanning manipulator, which is in fact a true limitation for scanning rough terrains.

The DYLEMA project is also committed to optimizing the task of mine location by means of a complete-coverage algorithm that ensures navigation autonomy while sweeping the whole infested area [9]. Real missions are accomplished by dividing the infested field into $n$ small areas of about $15 \mathrm{~m}$ by $15 \mathrm{~m}$. The DGPS antenna is located in a point that will be considered as the origin on the world reference frame for the entire field. The operator and the Operation Station are placed in a safe place. The SILO6 robot is located in a corner of the first area and its orientation with respect to the world reference frame is measured. After this calibration activity the robot scans the first area following the complete-coverage algorithm [9]. When the first area is fully scanned, the system database will report the map of potential alarms, which is the input for the deactivation team. This procedure is repeated for 


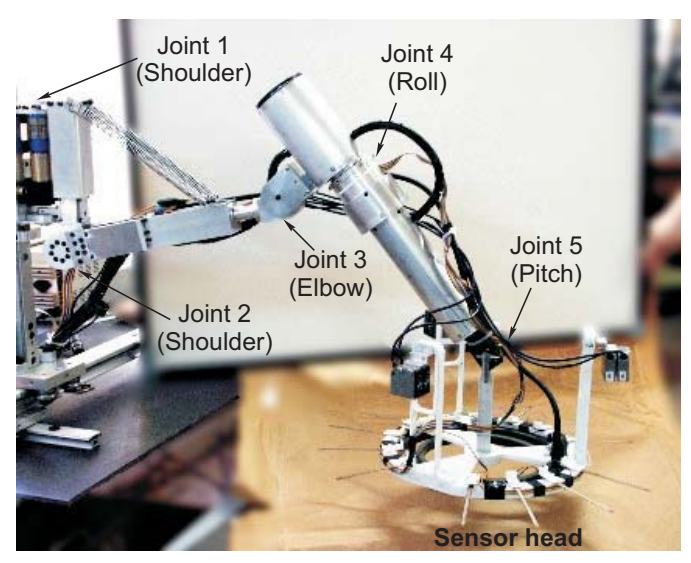

(a)

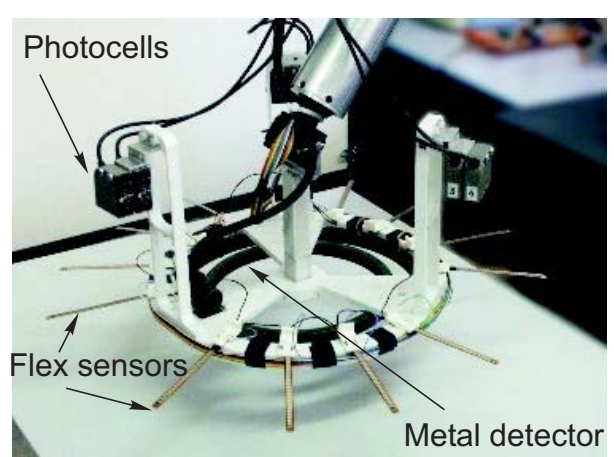

(b)

Fig. 2. Scanning system: a) Scanning manipulator; b) Sensor head

the rest of areas untill the complete scanning of the field.

\section{Scanning System}

The scanning system is designed to detect antipersonnel landmines while a mobile robot traverses the infected area. The scanning system may be broken down into the following subsystems, illustrated in Fig. 2(a):

\subsection{Sensor Head}

This subsystem contains a commercial mine detector and additional elements for detecting the ground and any objects in the way (see Figure 2(b)). The technologies available for detecting mines vary. The simplest one consists in a metal detector. However, it only detects mines with metallic parts, and it is inefficient at finding plastic mines. Detecting non-metallic mines calls for another type of sensor, such as sensors based on Ground Penetrating Radar (GPR) [10], chemical sensors [11] or artificial noses [12]. The DYLEMA project is devoted to the development of mobile robotic techniques for the identification and location of landmines. Sensor development does not fall within the scope of the project. Therefore, a metal detector is the simplest selection for a demining sensor, just to help in the detection and location of potential alarms. The Schiebel AN-19/2 commercial mine-detecting set was chosen for use in the DYLEMA project. This detector is in service in the US Army as well as in several NATO countries. It has been designed to detect typical mines with a very small proportion of metal content. To avoid interfering with the metal 
detector, the sensor head skeleton was made of Arnite (high-performance engineering plastic that combines high strength and rigidity).

The sensor head is configured to detect potential alarms, but also to allow the controller to maintain the sensor head at a given height above the ground (50-100 mm) using simple range sensors. These range sensors are photocells located in pairs defining the upper and lower limits of the band in which the sensor head works. This array allows the controller to estimate the ground plane and thus to adapt the sensor head to terrain irregularities. The type of photocell used (OMRON E3SA-DS50C43A) uses the direct reflex detection mode, which provides efficient detection in different surface types and colors. We tested the photocell on natural surfaces like sand, earth, concrete, stones and vegetation, under natural light, with a detection distance that was always over $200 \mathrm{~mm}$, which was enough for our purposes. The photocells were placed far enough from the metal detector $(150 \mathrm{~mm})$ to avoid false detection due to interference.

Touch sensors (flex sensors) were also installed to detect objects in the sensor head's trajectory, enabling the controller to steer around them. A flex sensor is a flexible-strip device whose electrical resistance changes when the strip is bent. These sensors are located around the sensor head as shown in Figure 2(b). When a flex sensor touches an object it bends, and its electrical resistance changes accordingly. By measuring the electrical current through its resistance, we can infer the contact. The flexibility of these devices makes for soft interaction between the sensor head and any objects in its way. The flex sensors were positioned at a radius that was also far enough from the center of the sensor head to avoid causing any interference with the metal detector.

\subsection{Scanning Manipulator}

The commercial mine detector is basically a local sensor. That means it is able to sense just one point. The efficiency of such a device can be improved by sweeping the sensor head through a large area. The easiest system would be a manipulator tailor-made for this task. Such a manipulator would require three DOFs for positioning the sensor in a 3D area; assuming that the system is scanning a non-flat area, motions in the $x, y$ and $z$ components would be required. Also, the sensor head would have to be adapted to small terrain inclinations; hence, two additional DOFs would be needed at the wrist to control detector attitude. The mine detector has radial symmetry, so no additional DOFs would be needed for orientation control. To sum up, a manipulator with at least five DOFs is needed to accomplish the task (see Fig. 2(a)). An RRR arm configuration is good enough for this application. Mobility is adequate and, because the links lie along a single vertical plane, there will be fewer 


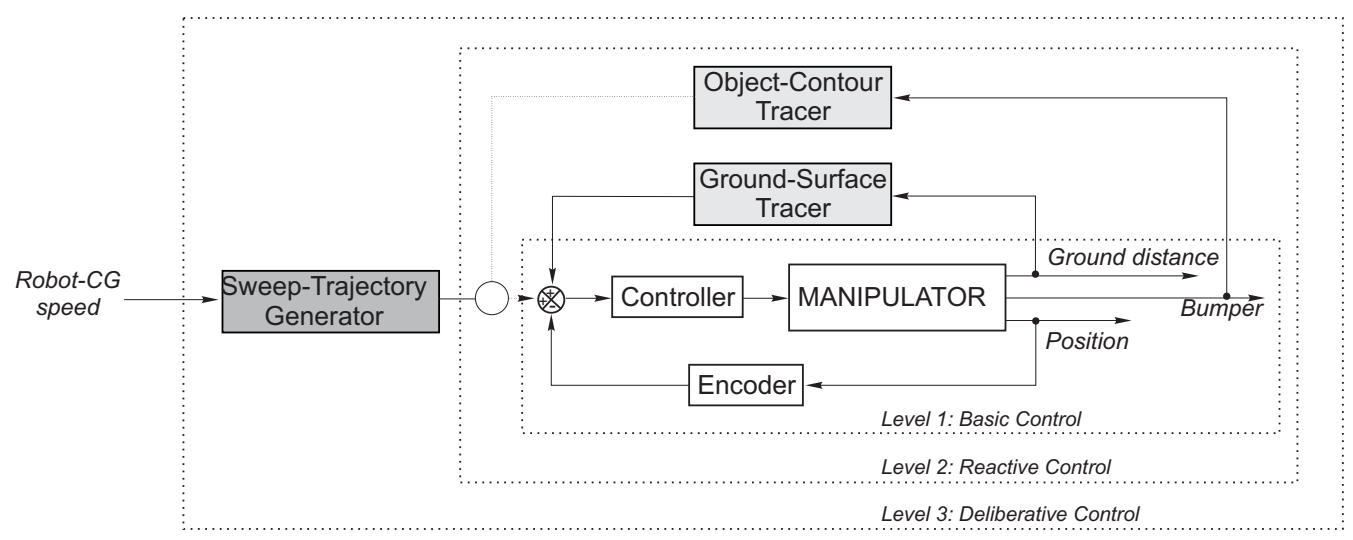

Fig. 3. The scanning-manipulator control architecture

collisions with the environment. The motors have been integrated inside the mechanical structure to decrease the volume swept by the manipulator structure when moving. This decreases the number of potential crushes between the manipulator and the environment.

\section{Control Architecture}

The scanning manipulator has to be controlled to seek for buried mines while it is being carried by a walking platform. To ensure efficient cooperation between the walking platform and the scanning system, both systems should navigate to achieve complete coverage of the minefield. Methods for complete coverage by mobile robots in unstructured environments already exist $[9,13]$. In this paper we propose a control architecture for the scanning system that achieves complete coverage of an unstructured environment when it is combined with the motion of the mobile platform that carries it. The control architecture of the scanning system is shown in Fig. 3.

The manipulator-control architecture is a deliberative/reactive hybrid. Apart from the basic joint controller, three modules govern manipulator motion, which are:

The sweep-trajectory generator: This is the deliberative module that generates the sweep trajectory to ensure complete coverage of the swept area.

The object-contour tracer: This is a reactive module that moves the sensor head around an obstacle using information from the bumper.

The ground-surface tracer: This is a reactive module that keeps the detector head at a constant distance from the ground, controlling its attitude as well. 


\subsection{Sweep-Trajectory Generator}

The sweep-trajectory generator computes the trajectory of the sensor head to ensure complete coverage of the swept area. This is done by means of a cross sweep, which is the most efficient way to scan for buried mines. It avoids overlapping scanned areas while ensuring complete coverage. The sensor-head trajectory with reference to the manipulator's base reference frame is depicted in Fig. 4(a). It scans an area that covers the width of the mobile robot that carries it $\left(2 y_{d}\right)$ and a length of $x_{d}$ along the $x$-axis. To coordinate the manipulator's motion with the walking robot's motion, certain conditions must be met:

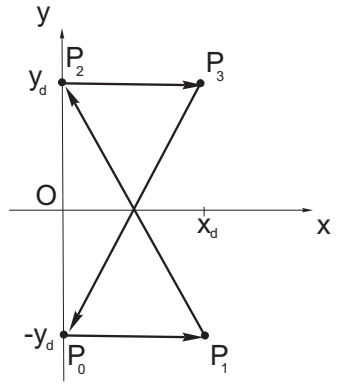

a)

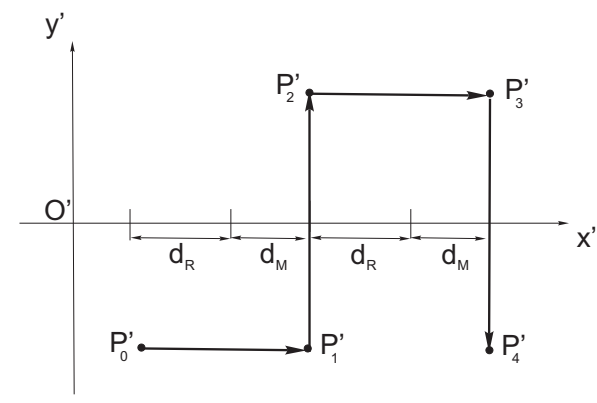

b)

Fig. 4. Cross-sweep trajectory: a) In the manipulator's reference frame; b) In the external reference frame

- The walking robot moves along the $x^{\prime}$ axis in an external reference frame $\left\{x^{\prime}, y^{\prime}\right\}$ at a constant speed of $v_{C G}$. The external reference frame is centered initially at the robot's CG position, and the $x^{\prime}$ axis lies along the robot's longitudinal axis.

- The manipulator's cross-sweep motion, combined with the motion of the robot's CG, results in zig-zag trajectories parallel to the $x^{\prime}$ and $y^{\prime}$ axes, as Fig. 4(b) shows.

- The complete manipulator motion takes place in a walking-robot gait-cycle time $\left(T_{c}\right)$.

To accomplish the first and second conditions, the diagonal span of the manipulator trajectory from $P_{1}$ to $P_{2}$ (see Fig. 4(a)) needs to travel back the same distance $x_{d}$ as traveled by the robot in the same interval $\left(t_{2}-t_{1}\right)$, where $t_{i}$ is the time it takes to reach $P_{i}$ (for $i=1 . .4$ ), that is:

$$
x_{d}=v_{C G}\left(t_{2}-t_{1}\right) .
$$

To accomplish the third condition, the time interval of each trajectory span needs to be a fraction of the robot's cycle time. Additionally, to ensure complete coverage of the swept area, the distance from $P_{0}^{\prime}$ to $P_{1}^{\prime}$ has to equal 


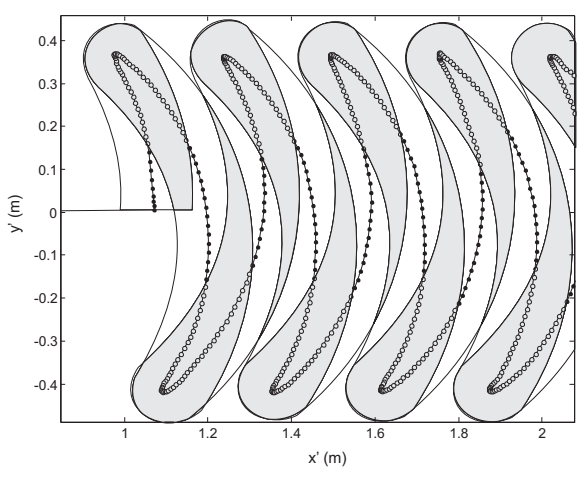

(a)

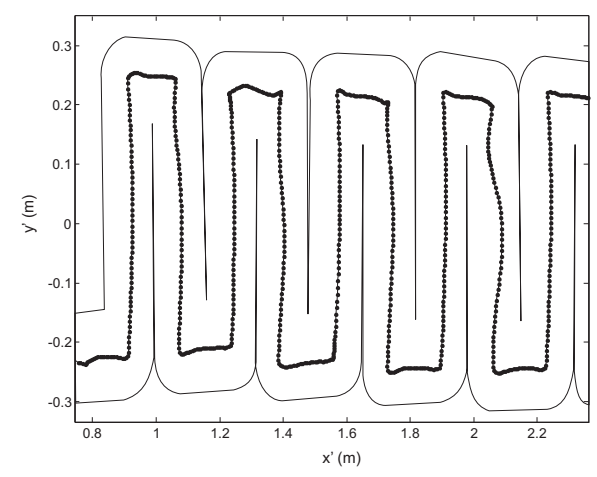

(b)

Fig. 5. Scanned area in the external reference frame using (a) a circular sweep; (b) a cross sweep

the sensor head's diameter. So we have to determine the time intervals of the trajectory spans so that:

$$
\operatorname{Dist}\left(P_{0}^{\prime}, P_{1}^{\prime}\right)=D
$$

where $D$ is the sensor head's diameter. Let us name $T_{1}=t_{1}-t_{0}$ and $T_{2}=t_{2}-t_{1}$. Let us also name $d_{R}$ the distance traveled by the walking robot during $T_{1}$ and $d_{M}$ the distance traveled by the manipulator during $T_{1}$. The problem equations are:

$$
\begin{aligned}
x_{d} & =v_{C G} T_{2} \\
D & =d_{R}+d_{M} \\
T_{c} & =2\left(T_{1}+T_{2}\right) .
\end{aligned}
$$

As a solution of this system of equations, we finally obtain the time intervals $T_{1}$ and $T_{2}$ that assure complete coverage of the scanned area:

$$
\begin{aligned}
T_{1} & =\left(1-\frac{x_{d}}{D}\right) \frac{T c}{2} \\
T_{2} & =\frac{x_{d}}{D} \frac{T_{c}}{2} .
\end{aligned}
$$

Now, the manipulator trajectory can be described in four steps, starting at $P_{0} \equiv\left(0,-y_{d}\right)$ : 
Step 1: Linear trajectory from $P_{0} \equiv\left(0,-y_{d}\right)$ to $P_{1} \equiv\left(x_{d},-y_{d}\right)$ in the $x$-direction, that is

$$
x=\frac{x_{d}}{T_{1}}\left(t-t_{0}\right) ; \quad y=-y_{d} .
$$

Step 2: Linear trajectory from $P_{1} \equiv\left(x_{d},-y_{d}\right)$ to $P_{2} \equiv\left(0, y_{d}\right)$ such that

$$
x=x_{d}-\frac{x_{d}}{T_{2}}\left(t-t_{1}\right) ; \quad y=-y_{d}+\frac{2 y_{d}}{T_{2}}\left(t-t_{1}\right) .
$$

Step 3: Linear trajectory from $P_{2} \equiv\left(0, y_{d}\right)$ to $P_{3} \equiv\left(x_{d}, y_{d}\right)$ in the $x$ direction, that is

$$
x=\frac{x_{d}}{T_{1}}\left(t-t_{2}\right) ; \quad y=y_{d}
$$

Step 4: Linear trajectory from $P_{3} \equiv\left(x_{d}, y_{d}\right)$ to $P_{0} \equiv\left(0,-y_{d}\right)$ such that

$$
x=x_{d}-\frac{x_{d}}{T_{2}}\left(t-t_{3}\right) ; y=y_{d}-\frac{2 y_{d}}{T_{2}}\left(t-t_{3}\right) .
$$

This cross-sweeping motion, combined with the motion over the robot's CG, results in zig-zag trajectories parallel to the robot's $x^{\prime}$ and $y^{\prime}$ axes in the external reference frame, as Fig. 4(b) shows. The cross-sweep trajectory is generated as a function of the speed of the walking robot's CG $\left(v_{C G}\right)$, and one cross sweep is performed per robot cycle. To show the advantages of applying the proposed sweep motion, a comparison between a circular sweep and a cross sweep has been performed. Figure 5(a) shows the experimental results of applying a complete-coverage circular sweep and compares them with the results of applying the cross sweep (Fig. 5(b)) using the SILO6 hexapod robot shown in Figure 1. The dotted line shows the trajectory of the sensor head's base center, while solid lines depict the total scanned area. As can be observed, in order to obtain complete coverage with a circular sweep, overlapping areas must exist (shaded areas in Fig. 5(a)), which make the method inefficient. However, the cross sweep generates complete-coverage trajectories without overlapping areas and is therefore demonstrated to be an efficient scanning method.

\subsection{Object-Contour Tracer}

An array of 12 flex sensors around the sensor head detects obstacles in the mine field (see Fig. 6). The reactive behavior of the object-contour tracer makes the manipulator move around the obstacle such that the touch sensors constantly detect the obstacle until the sweep path is found. 
Table 1

Motion commands for Virtual Detection Zones

\begin{tabular}{|c|c|c|c|}
\hline \multicolumn{4}{|c|}{ Span of sweep trajectory } \\
\hline \multicolumn{2}{|c|}{$P_{0}-P_{1}$} & \multicolumn{2}{|c|}{$P_{2}-P_{3}$} \\
& $P_{1}-P_{2}$ & \multicolumn{2}{c|}{$P_{3}-P_{0}$} \\
\hline VDZ & Motion direction & VDZ & Motion direction \\
\hline N & $-\mathrm{x}$ & $\mathrm{N}$ & $+\mathrm{x}$ \\
$\mathrm{NE}$ & $-\mathrm{x}+\mathrm{y}$ & $\mathrm{NE}$ & $+\mathrm{x}-\mathrm{y}$ \\
$\mathrm{E}$ & $+\mathrm{y}$ & $\mathrm{E}$ & $-\mathrm{y}$ \\
$\mathrm{SE}$ & $+\mathrm{x}+\mathrm{y}$ & $\mathrm{SE}$ & $-\mathrm{x}-\mathrm{y}$ \\
$\mathrm{S}$ & $+\mathrm{x}$ & $\mathrm{S}$ & $-\mathrm{x}$ \\
$\mathrm{SW}$ & $+\mathrm{x}-\mathrm{y}$ & $\mathrm{SW}$ & $-\mathrm{x}+\mathrm{y}$ \\
$\mathrm{W}$ & $-\mathrm{y}$ & $\mathrm{W}$ & $+\mathrm{y}$ \\
$\mathrm{NW}$ & $-\mathrm{x}-\mathrm{y}$ & $\mathrm{NW}$ & $+\mathrm{x}+\mathrm{y}$ \\
\hline
\end{tabular}

Because a 5-DOF manipulator cannot control the position and orientation of the bumpers in the sensor head, the object-contour tracer controls detection in the manipulator's base reference frame. This is done by defining eight virtual detection zones (VDZ) in the manipulator's base reference frame, which are North, North-East, East, South-East, South, South-West, West and NorthWest, such that the North is aligned with the positive direction of the $y$ axis of the manipulator's base reference frame (see Figure 7). Each time a flex sensor detects an object, we can know to which of these virtual zones it belongs, because we know the angular position $\alpha_{i}^{n}$ of each flex sensor $i(i=1 . .12)$ relative to the vector $\vec{n}$ of the end-effector, and by kinematics we also know the angular position of $\vec{n}$ relative to the $x$ axis of the manipulator's base reference frame $\alpha_{n}^{x}$. Thus, the angular position of flex sensor $i$ relative to the $x$ axis of the manipulator's base reference frame may be found by $\alpha_{i}^{x}=\alpha_{n}^{x}+\alpha_{i}^{n}$. Depending on the value of $\alpha_{i}^{x}$, we can know to which virtual detection zone the flex sensor belongs. The object-contour tracer orders motion in response to the detection of an object in one of the virtual zones. Table 1 shows the motion commands in response to detection in each virtual detection zone, so that the sensor head travels around the obstacle.

Figure 8(a) shows the cross-sweep trajectory with reference to the manipulator's base reference frame in an obstacle-free area. The two straight-line diagonal spans of the cross sweep have been changed to coordinated motion between two points, to lessen the CPUs computational burden and enable the reactive behaviors to succeed, but the efficiency of the cross motion remains the same. Figure 8(b) shows how the object-contour tracer modifies the sweep trajectory to avoid obstacles that stand in the way. 


\subsection{Ground-Surface Tracer}

While performing a cross-sweep motion and avoiding obstacles, the sensor head also adapts to the terrain profile reactively. Three pairs of photocells arrayed at $120^{\circ}$-degree intervals around the sensor head (see Fig. 9) measure the sensor head's distance to the ground. The sensor head's height above the ground is reactively controlled. Each pair of photocells defines a band of detection; one photocell has its detection threshold at the maximum distance allowable from the ground for detecting objects $(100 \mathrm{~mm})$, while the other photocell has its threshold at the minimum distance from the ground for avoiding ground contact $(50 \mathrm{~mm})$. Therefore, the ground-surface tracer commands motion up and down the vertical axis so that, in every pair of photocells, the first photocell detects nothing and the second detects ground to ensure that the sensor head remains inside the detection band. Figure 10 shows experimental results when the sensor head scans a stepped surface, shown in Figure 9. As this graph illustrates, the ground-surface-tracer adjusts the height of the scanning trajectory to follow the ground contour, while maintaining the horizontal cross-sweep motion.

Digital videos of the experiments described in this section are available at http://www.iai.csic.es/users/egarcia/coverage.htm.

\section{Conclusions}

The detection and location of antipersonnel landmines currently involves human operators handling manual equipment. However, the human community can obtain many benefits from the robotization of this activity. New sensors are required in order to detect landmines efficiently, but existing sensors can be carried by robots over infested fields.

This paper addresses the development of a manipulator that scans areas with a sensor head based on a metal detector and other sensors for scanning irregular ground in the presence of obstacles. The scanning system has been described, and the control architecture has been presented. This architecture enables the manipulator to adapt to terrain irregularities and avoid obstacles in a reactive manner, a clear improvement over the high cost of on-line terrain mapping. A new sweep method enabling the scanning manipulator to search efficiently for buried mines has also been proposed. The improvement obtained with the cross-sweep algorithm has been proved experimentally, as has the good performance of the reactive control for ground-contour following and obstacle avoidance. 
The scanning manipulator will contribute to the autonomous antipersonnellandmine detection process.

\section{Acknowledgements}

This work has been partially funded by the Spanish Ministry of Education and Science through Grants DPI2001-1595 and DPI2004-05824. The first author is supported by a postdoctoral CSIC-I3P contract granted by the European Social Fund.

\section{References}

[1] J.-D. Nicoud, Vehicles and robots for humanitarian demining, Industrial Robot 24 (2) (1997) 164-168.

[2] J. Trevelyan, Robots and landmines, Industrial Robot 24 (2) (1997) 114-125.

[3] E. Garcia, J. Estremera, P. Gonzalez de Santos, A control architecture for humanitarian-demining legged robots, in: Proc. Int. Conf. Climbing and Walking Robots, 2003, pp. 383-390, catania, Italy.

[4] P. Gonzalez de Santos, E. Garcia, J. Estremera, M. Armada, DYLEMA: Using walking robots for landmine detection and location, International Journal of Systems Science 36 (9) (2005) 545-558.

[5] H. Najjaran, A. Goldenberg, Landmine detection using an autonomous terrainscanning robot, Industrial Robot: An International Journal 32 (3) (2005) 240247 .

[6] M. Y. Rachkov, L. Marques, A. T. de Almeida, Multisensor demining robot, Autonomous Robots 18 (3) (2005) 275-291.

[7] H. Uchida, K. Nonami, Attitude control of a six-legged robot using sliding mode control, in: Proc. Int. Conf. Climbing and Walking Robots, 2003, pp. 103-110, catania, Italy.

[8] Y. Baudoin, Mobile robotic systems facing the humanitarian demining problem. State of the art, Tech. Rep. Task 3.1.4, International Test and Evaluation Program for Humanitarian Demining, The Royal Military Academy of Belgium (RMA) (December 2005).

[9] E. Garcia, P. Gonzalez de Santos, Mobile robot navigation with complete coverage of unstructured environments, Robotics and Autonomous Systems 46 (4) (2004) 195-204. 
[10] P. Gader, B. Nelson, H. Frigui, G. Vaillette, J. Keller, Landmine detection in ground penetrating radar using fuzzy logic, Signal Processing, Special Issue on Fuzzy Logic in Signal Processing 80 (6) (2000) 1069-1084.

[11] K. Albert, W. D.R., High-speed fluorescence detection of explosives-like vapour, Anal Chem 72 (9) (2000) 1947-1955.

[12] A. Rouhi, Land mines: Horrors begging for solutions, Chemical and engineering news 75 (10) (1997) 14-22.

[13] E. Acar, H. Choset, Robust sensor-based coverage of unstructured environments, in: Proc. International Conference on Intelligent Robots and Systems, 2001, pp. 61-68, Maui, Hawaii. 


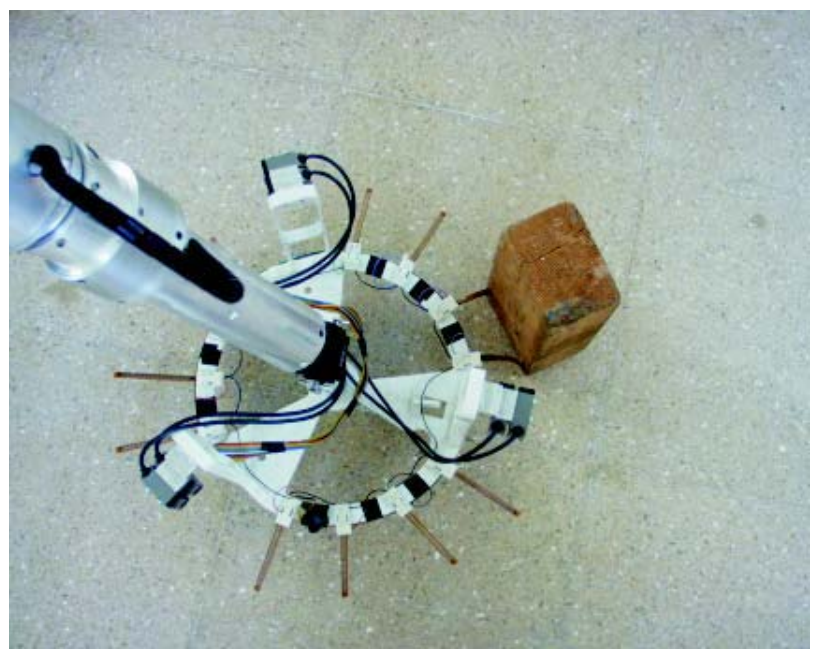

Fig. 6. Overhead view of experimental obstacle detection

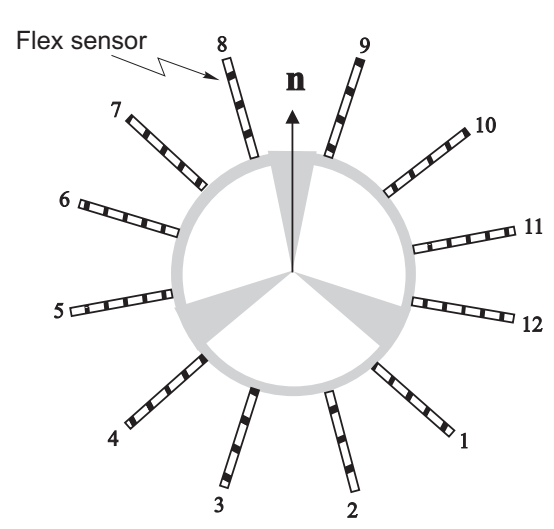

(a)

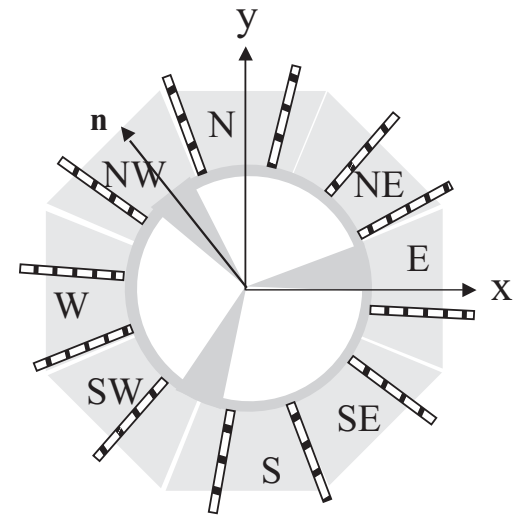

(b)

Fig. 7. (a) Distribution of flex sensors around the sensor head; (b) Virtual detection areas 


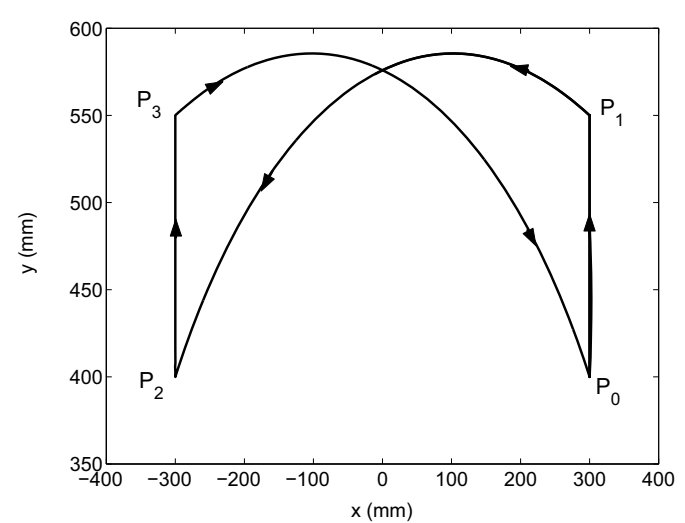

(a)

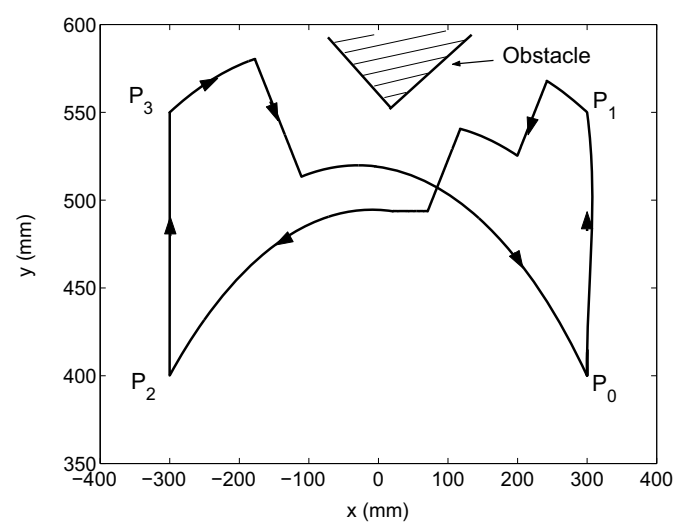

(b)

Fig. 8. (a) Cross-sweep trajectory in an obstacle-free area; (b) Sweep trajectory modified by object-contour tracer to avoid an obstacle (shaded area)

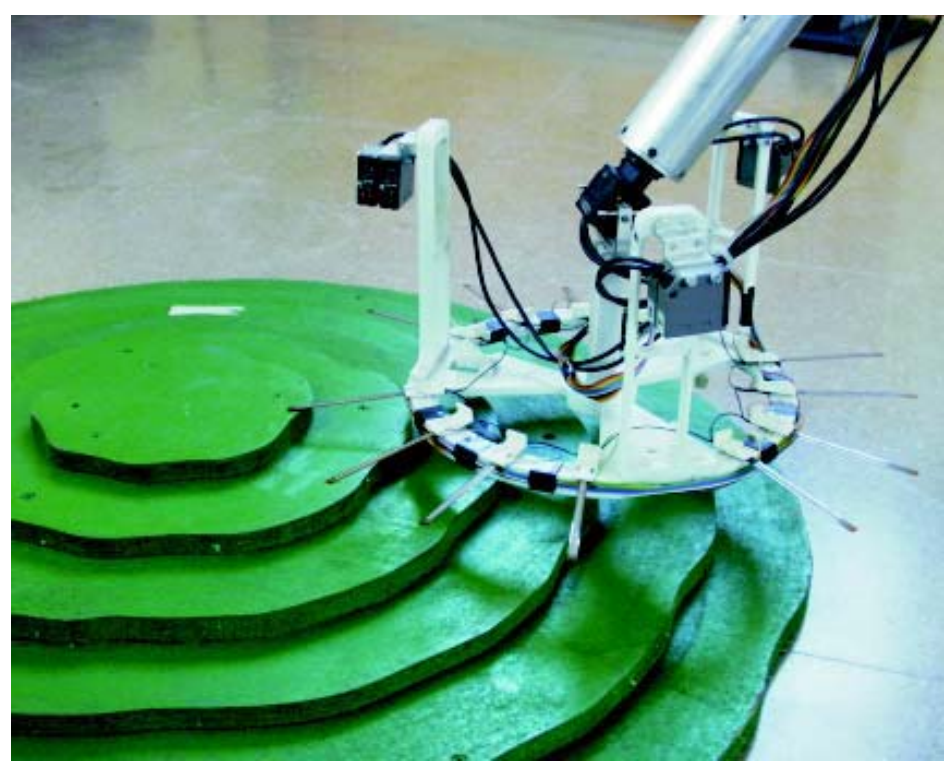

Fig. 9. Experimental ground adaptation 


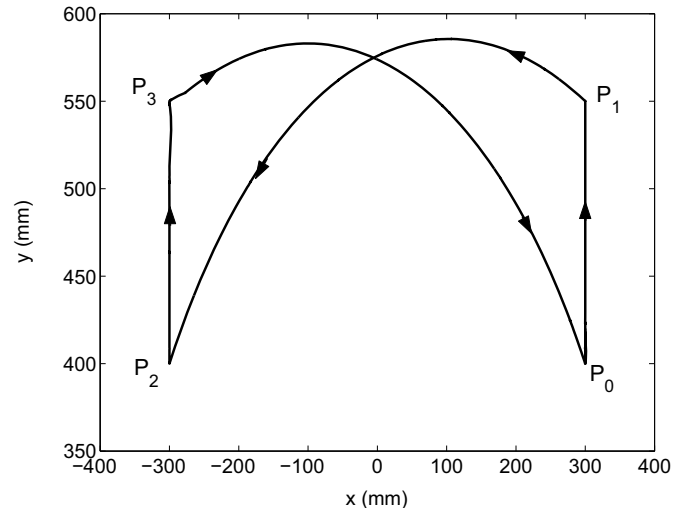

(a)

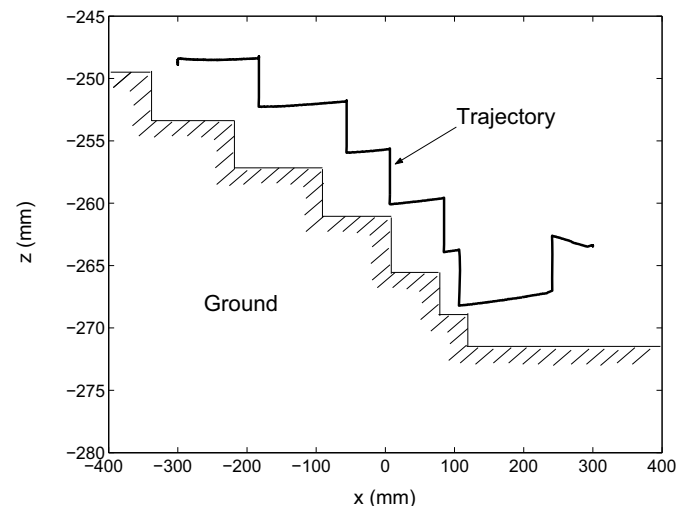

(b)

Fig. 10. Cross sweep trajectory on stepped ground (shaded area) (a) $X Y$ plane; (b) $X Z$ plane 